\title{
Evaluating Performance of the HIPERLAN CAC Layer Protocol for Asynchronous Traffic
}

\author{
Constantine Coutras \\ Department of Computer Science \\ Illinois Institute of Technology \\ 10 West 31st Street \\ Chicago, IL 60616 \\ email:coutcon@cs.iit.edu
}

\author{
Peng-Jun Wan \\ Department of Computer Science \\ Illinois Institute of Technology \\ 10 West 31st Street \\ Chicago, IL 60616 \\ email:wan@cs.iit.edu
}

\begin{abstract}
The growing interest in wireless systems and networks has led to the first Wireless LAN (WLAN) protocols. The Medium Access Control (MAC) layer protocols of such protocol suites are of key importance. The Radio Equipment and Systems (RES) Technical Committee of the European Telecommunications Standards Institute has proposed the High PErformance Radio LAN (HIPERLAN) protocol suite. In this paper we present, study, analyze and evaluate the performance of the MAC layer of the HIPERLAN protocol suite for asynchronous data transfer. Analytical models that take into account the phenomena of hidden nodes and capture are presented during the analysis. Numerical results from both analysis and simulation are presented, so that the issues involved are better understood.
\end{abstract}

\section{Introduction}

In recent years we are witnessing a trend of moving from tethered systems to wireless systems. This is made possible due to the ongoing evolution of wireless systems in the last decade.

Wireless LANs (WLANs) are part of this evolution. New protocols for the Physical and Data Link layers are needed due to the characteristics of the wireless medium. It is desired that the new protocol suites keep the wireless characteristic as transparent as possible to the upper layers so that they do not need to go through major changes.

The wireless medium differs in some very important ways: 1)The bit error rate is much higher in the air than in a wired medium. 2)When a node transmits a packet it can not be certain if the receiver received successfully, thus an acknowledgment is required. 3)It is possible that at some point in time two (or more) nodes do not have direct access to each other in which case we say that they are hidden from each other and no successful transmission can take place directly between them. 4)It is possible for a receiver to receive two partially or completely overlapping in time packets and still be able to distinguish and successfully receive one of them, basically due to different signal power levels. This is known as the phenomenon of capture.

So far two protocol suites have been proposed for WLANs. The first is the result of the work done by IEEE committee 802.11 and has recently become an official standard. Studies of the protocol for asynchronous data traffic can be found in [4] and [3], while studies of time-bounded data traffic can be found in [6]. The second protocol is currently under development by the European Telecommunications Standards Institute (ETSI) and is named High PErformance Radio Local Area Network (HIPERLAN). The HIPERLAN protocol functionality is presented in [7]. Other sources of information and analysis of the HIPERLAN protocol can be found in [1], [2], [8], [9], [10], [12] and [11]. No analytical models that take into account the phenomena of hidden nodes and capture are presented in these papers, although a first attempt to gather simulation results considering only the possibility of hidden nodes can be found in [8] and [12]. In [12] an attempt is also made to provide an analytical model for HIPERLAN taking into account only the phenomenon of hidden nodes. The result is an upper bound on performance that is based on the following assumption: if a node is hidden from a group of nodes, then all other nodes that are not hidden from that node are also hidden from that same group of nodes, and the nodes in both groups are not hidden from each other. This assumption, of course, is a special case, far from the general case. In this paper we provide analysis considering both the phenomena of hidden nodes and capture. We first analyze the effect of hidden nodes on the Elimination-Yield Non-preemptive Priority Multiple Access (EY-NPMA) mechanism 
for channel access and then the effect of both the phenomena of hidden nodes and capture on the performance of the HIPERLAN Channel Access Control (CAC) Layer protocol. Analytical and simulation results are presented.

\section{Overview of the HIPERLAN CAC Layer Protocol}

The CAC layer is actually the "lower sublayer" of the MAC layer that basically deals with the mechanism of accessing the channel (EY-NPMA mechanism).

The EY-NPMA mechanism is an access mechanism with three phases. The three phases of the EY-NPMA mechanism constitute the contention phase of the Synchronized Channel Access Cycle.

In Figure 1 we see a renewal interval, its components, and their components as well. Transmission is denoted by black color, while its absence is denoted by white color, and a different shade filling is used for the synchronization slot. All three phases of the EY-NPMA mechanism are divided into time slots, which are shown as rectangular boxes.

Renewal Interval

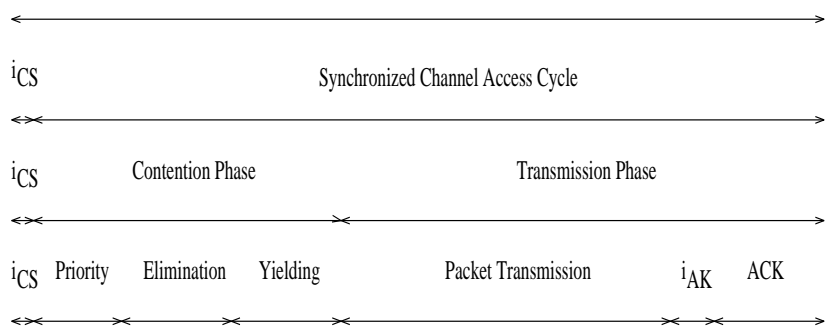

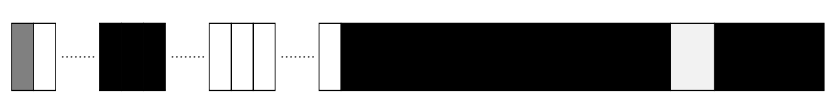

\section{Figure 1. The EY-NPMA mechanism.}

We will refer to a node that wishes to access the channel as an active node and $A(n)$ and $I(n)$ will denote the sets of active and inactive nodes of priority $n$.

In the prioritization phase, an active node of priority $n$ must signal its intention to access the channel by transmitting a burst during the $n t h$ time slot, provided that no active nodes of higher priority have already signaled their intention to access the channel. We are assuming a total of $m_{\mathrm{CP}}$ priority levels, from 0 to $m_{\mathrm{CP}}-1$. As soon as an active node has transmitted a burst, this phase is over and the next phase starts.

In the elimination phase each active node bursts a signal for a random number of time slots and then listens to the channel; if another active node is still bursting this active node is eliminated, otherwise it may continue into the yielding phase. In the elimination phase we have a maximum of $m_{\mathrm{ES}}$ elimination slots. The probability of bursting in an elimination slot is $p_{\mathrm{E}}$. The maximum burst allowed is $m_{\mathrm{ES}}-1$ time slots.

In the yielding phase each active node listens for a random number of time slots and then, if the channel is still free, starts a packet transmission. In the yielding phase we have a maximum of $m_{Y S}$ yielding slots. The probability of yielding in a yielding slot is $p_{\mathrm{Y}}$. An active node can listen for a maximum of $m_{Y S}-1$ time slots.

\section{Channel Access in the Presence of Hidden Nodes}

The following assumptions simplify the development of the analytical framework for our study:

- The effect of packet errors due to bit errors is ignored.

- Due to the high channel speed used in HIPERLAN we can assume relatively limited node mobility. This means that if two nodes are hidden from each other in the beginning of a renewal interval they will remain hidden through out that renewal interval.

During the prioritization phase, for an active node of lower priority to falsely determine that it may continue into the next phase, it will have to be hidden from all active nodes of higher priorities.

An active node might falsely determine that it has survived the elimination phase if it is hidden from all active nodes that burst for more time slots. A simple example is illustrated in Figure 2, where we see nine active nodes. Lets assume that nodes 1, 2, 3, 4 and 5 are not hidden from each other and nodes $6,7,8$ and 9 are also not hidden from each other. And all nodes in one group are hidden from all nodes in the other, with one exception. Nodes 4 and 6 are not hidden from each other. If after the elimination phase is over nodes 1,3 and 4 have bursted for the same number of slots, nodes 1 and 3 will determine that they have survived, while for node 4 it depends on what node 6 has done. If 6 has bursted for more slots than 4 , then 4 will be eliminated thus nodes 1 and 3 falsely determined survival- otherwise it will continue. If 6 bursted the same number of slots as 9 and more than 7 and 8 , then 9 will survive but 6 will only if it did not get eliminated by 4 . If 6 has bursted for the same number of slots as 4 , then all nodes $1,3,4,6$ and 9 determine correctly that they survived the elimination phase. In case that 4 continues but not 6 or vice-versa, we will have two mutually exclusive groups of contenders continuing in the next phase. But if both 4 and 6 continue, then two non mutual exclusive groups continue, one consisting of 1,3 and 4 and the other of 6 and 9 . 


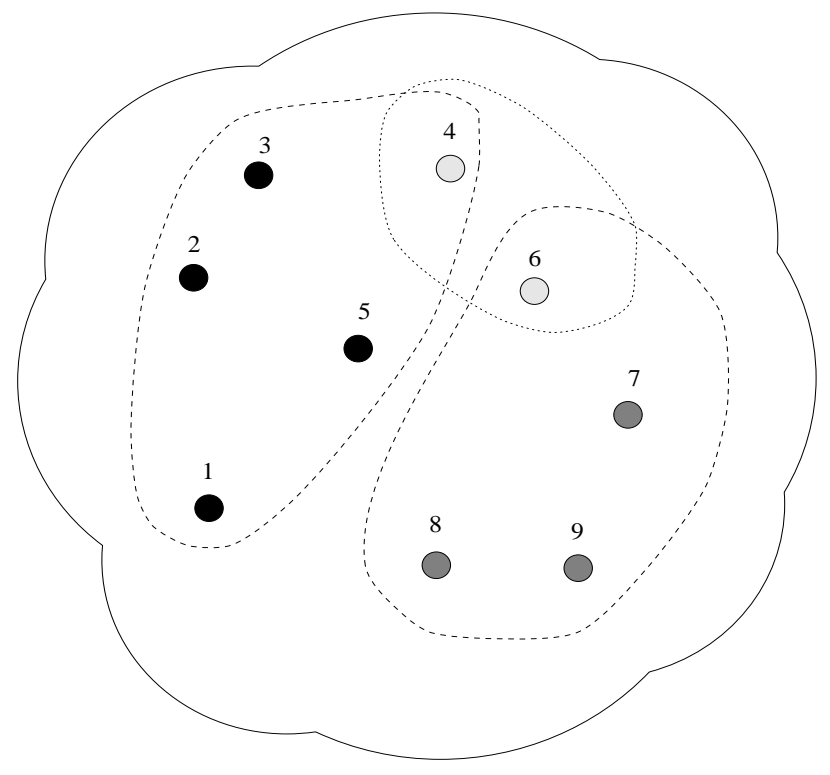

Figure 2. Groups of contending nodes

In the yielding phase, if the groups of remaining nodes that are not hidden from each other, are mutually exclusive, then for each such group the best performer(s) will transmit. But if these groups are not mutually exclusive a node might falsely determine channel access. To continue the previous example, lets assume that both 4 and 6 survived the elimination phase. During the yielding phase since node 1 "sees" nodes 3 and 4 it will have to do better than them to access the channel. Node 4 will also be depended on node 6 , which in turn will depend on nodes 4 and 9. It is possible for both 1 and 6 to access the channel without having the same performance.

If during the elimination phase an active node $i$ eliminates a group of active nodes that are hidden from any other surviving active node, and then during the yielding phase does not win channel access, the nodes that lost during the elimination phase to $i$ will see no transmission. In this case they are allowed to transmit their packet under the hidden elimination condition.

We now calculate the probability of accessing the channel for an active node $i$ that is not hidden from $c-1$ other active nodes of its own priority, with which it forms a group of competitors (from its own point of view) as described in the previous section.

Let $C_{n}$ be the random variable that represents the number of active nodes of priority $n$ (specifically including $i$ ) that are competing for the channel, and let $T$ be the random variable that represents the total number of nodes that the first node sees as winners (including it self). Let $X_{\mathrm{E}}$ be the random variable that represents the number of elimination slots that a node will be bursting in during the elimina- tion phase and $X_{\mathrm{Y}}$ be the random variable that represents the number of yielding slots that a node will be listening in during the yielding phase. Since the random variables are geometrically distributed, we have :

$$
\begin{gathered}
P\left\{X_{\mathrm{E}}=x\right\}= \begin{cases}p_{\mathrm{E}}^{x}\left(1-p_{\mathrm{E}}\right) & \text { if } x<m_{\mathrm{ES}}-1 \\
p_{\mathrm{E}}^{x} & \text { if } x=m_{\mathrm{ES}}-1\end{cases} \\
P\left\{X_{\mathrm{Y}}=x\right\}= \begin{cases}p_{\mathrm{Y}}^{x}\left(1-p_{\mathrm{Y}}\right) & \text { if } x<m_{\mathrm{YS}}-1 \\
p_{\mathrm{Y}}^{x} & \text { if } x=m_{\mathrm{YS}}-1\end{cases}
\end{gathered}
$$

Let $E$ be the random variable that represents the number of nodes that survive the elimination phase and $Y$ be the random variable that represents the number of nodes that survive the yielding phase.

In the absence of hidden nodes we have

$$
\begin{aligned}
& P\left\{T=t \mid C_{n}=c\right\}= \\
& \sum_{j=t}^{c}\left(\begin{array}{c}
c-1 \\
j-1
\end{array}\right) P\left\{E=j \mid C_{n}=c\right\}\left(\begin{array}{c}
j-1 \\
t-1
\end{array}\right) P\{Y=t \mid E=j\}
\end{aligned}
$$

where

$$
\begin{aligned}
& P\left\{E=j \mid C_{n}=c\right\}= \\
& \sum_{k=1}^{m_{E S}-1} P\left\{X_{\mathrm{E}}=k\right\}^{j} P\left\{X_{\mathrm{E}}<k\right\}^{(c-j)}
\end{aligned}
$$

and

$$
\begin{aligned}
& P\{Y=t \mid E=j\}= \\
& \quad \sum_{w=0}^{m_{Y S}-2} P\left\{X_{\mathrm{Y}}=w\right\}^{t} P\left\{X_{\mathrm{Y}}>w\right\}^{(j-t)}
\end{aligned}
$$

After the elimination phase is over, it is possible that not all nodes, which a node $i$ "sees" winning the elimination phase, will continue into the next phase. This is due to the fact that some of them could have been eliminated by other -non hidden from them- nodes, that are hidden from node $i$, as we have seen in the previous section. It is also possible that during the yielding phase, even more nodes are eliminated before $i$ wins channel access due to the fact that they hear transmission from other nodes not hidden from them but -again- hidden from $i$. Lets refer to these nodes that eliminate nodes that $i$ does not see being eliminated during the two phases as "helper" nodes to node $i$.

In order to calculate an exact probability of accessing the channel for a node $i$ under the effect of hidden nodes we would have to account for every possible hidden node 
pair in the WLAN. But since the calculation of the probability of channel access under no hidden nodes is actually a calculation of the "maximum effort" required by a node to determine that it won channel access, a lower bound can be calculated using the equation for the case of no hidden nodes. We need to examine how far from the actual value this approximation can be.

When calculating the probability of channel access for node $i$, using the equation for no hidden nodes we are not taking into account the effect that the "helper" nodes to node $i$ have on this calculation. So it is obvious that how close the above mentioned lower bound will be to the actual value depends on the number of these "helper" nodes. It is very important to state that if a "helper" node $k$ does not win channel access it has no effect on the calculation of the probability of channel access for $i$. This of course is due to the hidden elimination condition. In the next section, we will argue that the number of "helper" nodes $k$ are extremely minimized under the total conditions necessary for a successful transmission over the channel, thus this approximation is a very good one for calculating the final throughput.

\section{Packet Transmission in the Presence of Hid- den Nodes and Capture}

Let $d_{i j}$ denote the distance between two nodes $i$ and $j$. A transmission by station $i$ will be "captured" by station $j$ if no other node in a circle of radius $\alpha d_{i j}, \alpha \geq 1$, around $j$ is transmitting simultaneously. The parameter $\alpha$ will be referred to as the capture parameter and the area surrounding node $j$ of radius $\alpha d_{i j}$ will be referred to as $j^{\prime} s$ capture area for node $i$. Let's further denote $A_{j i}(\alpha)$ as the set of nodes that are in $j^{\prime} s$ capture area for $i$, and $\bar{A}_{j i}(\alpha)$ the set of nodes that are out of $j^{\prime} s$ capture area for $i$.

We extend the assumption of relatively limited node mobility so that if a node is inside or outside of a specific capture area it will remain so throughout the renewal interval.

In Figure 3 we see a WLAN that consists of a number of nodes that have determined channel access (triangular with black filling) and their corresponding destination nodes (circles with black filling). The receiver's capture area for the specific transmitter is a continuous circle, while the transmitter's capture area for the specific receiver sending back an acknowledgment is a dotted circle.

In order for a node to successfully transmit a packet and successfully receive its acknowledgment, these conditions have to be met:

- The transmitter and receiver nodes are not hidden from each other.

- After the contention phase is over, the transmitter node determines survival, while the receiver node, if active, does not.

- If nodes other than the transmitter node have survived the contention phase, they have to be either hidden from the receiver node or outside of the receivers capture area for the transmitter so that the transmitter's packet can be successfully received.

- No node that is not hidden from the transmitter or that is not outside of the transmitter's capture area for the receiver, receives successfully a packet. This guarantees that if the transmitter has successfully transmitted a packet he will also successfully receive its acknowledgment.

From the conditions stated above, for a "helper" (to node $i$ ) node $k$, to "assist" node $i$ in eliminating nodes that are competing with $i$ for channel access, it should also be hidden from node $j$ or out of $j^{\prime} s$ capture area for node $i$, it should also transmit to a node that is hidden from $i$ or out of $i$ 's capture area for $j$, or to a node that does not successfully receive a packet. Having this fact in mind and the fact that values of $p_{h}$ are usually less than 0.10 , we are expecting that using $P\left\{T=t \mid C_{n}=c\right\}$ as calculated for the no hidden nodes and no capture case will result in a very good approximation of $P\left\{T=t \mid C_{n}=c\right\}$ and consequently of $P_{i j}$.

We now give an example of how these conditions are met or not in Figure 3.

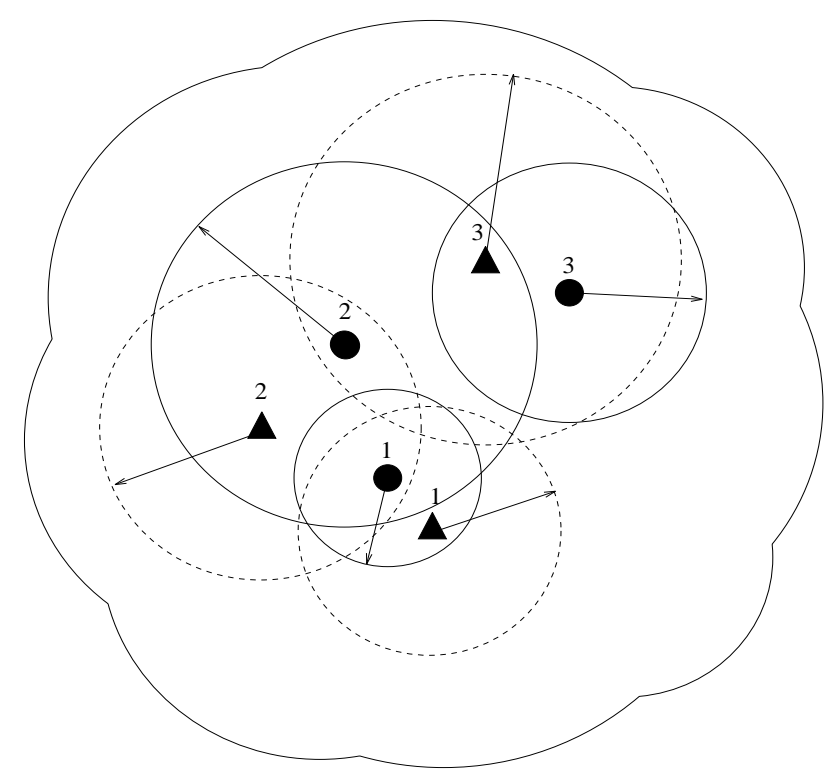

Figure 3. Hidden nodes and Capture Areas

In Figure 3 we see that for receiver nodes 1 and 3 no interfering node is in their capture areas for their transmitters. These nodes will be able to successfully receive a 
packet from their transmitters as long as they are not hidden from them. But for receiver node 2 to successfully receive a packet from transmitter node 2 , it has to be hidden from transmitter node 3 . After the transmission of the packets, the receiver nodes that successfully received a packet will transmit an acknowledgment. For transmitter node 1 there is no problem. The case is different for transmitter nodes 2 and 3 . In transmitter node 2's capture area are both receiver nodes 1 and 2 . Since receiver node 1 successfully received a packet from transmitter node 1 and sent back an acknowledgment, transmitter node 2 will receive this transmission and will not be able to distinguish it from the possible transmission of an acknowledgment sent by receiver node 2 , if receiver node 2 was hidden from transmitter node 3 as we saw previously. So in no case will there be a successful transmission between the nodes of pair 2 . If transmitter node 3 and receiver node 2 are hidden, then transmitter node 3 will successfully receive the acknowledgment from receiver node 3 . Also if transmitter node 3 and receiver node 2 are not hidden, then receiver node 2 will not be sending an acknowledgment since it did not successfully receive a packet as we saw previously, so in this case transmitter node 3 will be able to successfully receive the acknowledgment from receiver node 3 . In this example we have two successful transmissions at the same time for a renewal interval.

\section{Throughput Analysis}

We will assume that all sources -of any priority- generate packets following the Poisson distribution. We will further assume that the total offered load by each priority (new arrivals plus retransmission attempts) also follows the Poisson distribution. The arrival rate of packets that are from node $i$ and are destined for node $j$ is $\lambda_{i j}$, with the total arrival rate of packets of priority $n$ being $\lambda(n)$, and the overall total arrival rate for the system being $\lambda$. The offered load (arrivals plus retransmission) for source $i$ that is destined for node $j$ is $G_{i j}$, with the total offered load for all nodes that are of priority $n$ being $G(n)$, and the overall total load for the system being $G$. In a stable system we want the throughput from one node to another to be equal to the arrival rate of packets from that node to another, otherwise if the arrival rate is greater the node will soon be overflowed and packets lost due to finite storage capacity at each node.

The total throughput for the system is:

$$
S=\sum_{n=0}^{m_{\mathrm{CP}}-1} S(n)
$$

And the total throughput for each priority is:

$$
S(n)=\sum_{i=1}^{T(n)} \sum_{j=1}^{(\text {Total }-1)} S_{i j} \text { for } i \neq j
$$

where $T(n)$ is the total number of nodes for priority $n$ and Total is the total number of nodes in the system. The throughput from node $i$ to node $j$ is :

$$
S_{i j}=\alpha_{i} G_{i j} P_{i j}
$$

where $P_{i j}$ is the probability of a successful transmission from node $i$ of priority $n$ to node $j$ and $\alpha_{i}$ is the percentage of the offered load from $i\left(G_{i}\right)$ that gets to compete. The value of $\alpha_{i}$ is the total number of packets eligible to compete over the total number generated in a renewal interval, so for our Poisson traffic model:

$\alpha_{i}=\frac{\sum_{k=1}^{\infty} \frac{\left(G_{k} E\left(T_{\mathrm{S}}\right)\right)^{k}}{k !} e^{-\left(G_{k} E\left(T_{\mathrm{S}}\right)\right)}}{\sum_{k=1}^{\infty} k \frac{\left(G_{k} E\left(T_{\mathrm{S}}\right)\right)^{k}}{k !} e^{-\left(G_{k} E\left(T_{\mathrm{S}}\right)\right)}}=\frac{1-e^{-\left(G_{k} E\left(T_{\mathrm{S}}\right)\right)}}{G_{k} E\left(T_{\mathrm{S}}\right)}$

where $E\left(T_{\mathrm{S}}\right)$ is the expected value of the renewal interval $T_{\mathrm{S}}$.

The probability of success $P_{i j}$ is based on satisfying the conditions stated in the previous section and as we will see becomes tedious.

Let $H_{n}$ be the random variable that represents the number of packets of priority higher that $n$. The probability of non-existing packets of higher priority in a time interval equal to the expected renewal interval is:

$$
P\left\{H_{n}=0\right\}=\prod_{k=0}^{(n-1)} e^{-G(k) E\left(T_{\mathrm{S}}\right)}
$$

In the absence of hidden nodes and capture the probability of success $P_{i j}$ if $i$ is of priority $n$ is:

$$
P_{i j}=P\left\{H_{n}=0\right\} \sum_{c=1}^{T(n)} P\left\{C_{n}=c\right\} P\left\{T=1 \mid C_{n}=c\right\}
$$

In order to calculate the probability of success $P_{i j}$ under the presence of hidden nodes and capture, we first need to introduce some random variables: $X_{\mathrm{T}}$ represents the number of transmitters, other than $i$, that are not hidden from $i$ that have also detected that they have won channel access and are not hidden from $j$ and are not out of $j$ 's capture area for $i ; X_{\mathrm{W}}$ represents the number of active nodes that are hidden from $i$ that have also detected that they have won channel access and are not hidden from $j$ and are not 
out of $j$ 's capture area for $i ; X_{\mathrm{RT}}$ represents the number of receivers that have successfully received a packet from a transmitting node not hidden from $i$, and are not hidden from $i$ and are not out of $i$ 's capture area for $j$; $X_{\mathrm{RW}}$ represents the number of receivers that have successfully received a packet from a transmitting node hidden from $i$, and are not hidden from $i$ and are not out of $i$ 's capture area for $j ; W_{i}$ represents the number of active nodes that are hidden from node $i ; W_{i j}$ represents the number of active nodes that are hidden from node $i$, but are not hidden from node $j$, and $T_{j}$ represents the number of active nodes that are not hidden from $i$ and are wining channel access concurrently with $i$ and are not hidden from node $j$.

We first calculate the probability of success $P_{i j}$ if $i$ and $j$ are of the same priority:

$$
\begin{aligned}
& P_{i j}=\left(1-p_{h}\right) P\left\{H_{n}=0\right\} \\
&\left(\sum_{c=2}^{T(n)} P\left\{C_{n}=c \mid j \in T(n) \cap A(n)\right\}\right. \\
& \sum_{w=0}^{c-2} \sum_{t=1}^{c-w} P\left\{W_{i}=w \mid j \in T(n) \cap A(n), C_{n}=c\right\} \\
& P\left\{T=t \mid C_{n}=(c-w)\right\} \frac{(c-w)-t}{(c-w)-1} \\
& P\left\{X_{\mathrm{T}}=0 \cap X_{\mathrm{RT}}=0 \cap X_{\mathrm{W}}=0 \cap X_{\mathrm{RW}}=0\right. \\
&+\sum_{c=1}^{T(n)} P\left\{C_{n}=c \mid j \in T(n) \cap I(n)\right\} \\
& \sum_{w=0}^{c-1} \sum_{t=1}^{c-w} P\left\{W_{i}=w \mid j \in T(n) \cap I(n), C_{n}=c\right\} \\
& P\left\{T=t \mid C_{n}=(c-w)\right\} \\
& P\left\{X_{\mathrm{T}}=0 \cap X_{\mathrm{RT}}=0 \cap X_{\mathrm{W}}=0 \cap X_{\mathrm{RW}}=0\right. \\
&\left.\left.T=t, W_{i}=w, C_{n}=c\right\}\right)
\end{aligned}
$$

where $\frac{(c-w)-t}{(c-w)-1}$ insures that $j$ has not won channel access, and :

$$
\begin{gathered}
P\left\{W_{i}=w \mid j \in T(n) \cap A(n), C_{n}=c\right\}= \\
\left(\begin{array}{c}
c-2 \\
w
\end{array}\right)\left(p_{h}\right)^{w}\left(1-p_{h}\right)^{(c-1-w)}
\end{gathered}
$$

and:

$$
\begin{aligned}
& P\left\{W_{i}=w \mid j \in T(n)\right.\left.\cap I(n), C_{n}=c\right\}= \\
&\left(\begin{array}{c}
c-1 \\
w
\end{array}\right)\left(p_{h}\right)^{w}\left(1-p_{h}\right)^{(c-1-w)}
\end{aligned}
$$

If $i$ and $j$ are not of the same priority, the calculation of the probability of success $P_{i j}$ is simplified to:

$$
\begin{aligned}
P_{i j}= & \left(1-p_{h}\right) P\left\{H_{n}=0\right\} \\
& \sum_{c=1}^{T(n)} P\left\{C_{n}=c\right\} \sum_{w=0}^{c-1} \sum_{t=1}^{c-w} P\left\{W_{i}=w \mid C_{n}=c\right\} \\
& P\left\{T=t \mid C_{n}=(c-w)\right\} \\
& P\left\{X_{\mathrm{T}}=0 \cap X_{\mathrm{RT}}=0 \cap X_{\mathrm{W}}=0 \cap X_{\mathrm{RW}}=0 \mid\right. \\
& \left.T=t, W_{i}=w, C_{n}=c\right\}
\end{aligned}
$$

In order to calculate accurately the probability of $X_{\mathrm{RT}}=$ 0 or $X_{\mathrm{RW}}=0$ we would have to take into account the probability of a non transmission to a receiver in $i^{\prime} s$ capture area for $j$, but also the probability of a transmission to a receiver in $i^{\prime} s$ capture area for $j$ that is not successful due to a collision. We will be taking into account the probability of a non transmission to a receiver in $i^{\prime} s$ capture area for $j$, but we will be approximating the probability of a non successful transmission to a receiver in $i^{\prime} s$ capture area for $j$ with the probability of more than one transmissions to that receiver, thus approximating $P\left\{X_{\mathrm{RT}} \neq 0\right\}$ and $P\left\{X_{\mathrm{RW}} \neq 0\right\}$ with the probability that a receiver in $i^{\prime} s$ capture area for $j$ only receives one transmission. This of course is an approximation since the receiver could also receive from another source but distinguish transmission due to the effect of capture. This is the only additional approximation introduced in our calculations. This approximation allows us to calculate $P\left\{X_{\mathrm{RT}}=0\right\}$ and $P\left\{X_{\mathrm{RW}}=0\right\}$ together and we will denote $P\left\{X_{\mathrm{RT}}=0 \cap X_{\mathrm{RW}}=0\right\}$ as $P\left\{X_{\mathrm{R}}=0 \mid p\right\}$ where $p$ is the number of total sources that have determined that they have won channel access, other than nodes $i$ and $j$. This approximation will be the best for $a \rightarrow \infty$ and the worst for $a \rightarrow 1.0$, because as $a \rightarrow \infty$, we have: $X_{\mathrm{RT}} \rightarrow 0$ and $X_{\mathrm{RW}} \rightarrow 0$. Of course in the absence of the phenomenon of capture we have: $P\left\{X_{\mathrm{RT}}=0\right\}=P\left\{X_{\mathrm{RW}}=0\right\}=1$.

We now calculate the probability that the conditions stated in the previous section for a successful transmission are satisfied:

$$
\begin{gathered}
P\left\{X_{\mathrm{T}}=0 \cap X_{\mathrm{RT}}=0 \cap X_{\mathrm{W}}=0 \cap X_{\mathrm{RW}}=0 \mid\right. \\
\left.T=t, W_{i}=w, C_{n}=c\right\}= \\
\sum_{t_{j}=0}^{t-1}\left(\begin{array}{c}
t-1 \\
t_{j}
\end{array}\right)\left(1-p_{h}\right)^{t_{j}} p_{h}^{\left(t-1-t_{j}\right)} \\
\sum_{w_{i j}=0}^{w}\left(\begin{array}{c}
w \\
w_{i j}
\end{array}\right)\left(1-p_{h}\right)^{w_{i j}} p_{h}^{\left(w-w_{i j}\right)} \\
P\left\{X_{\mathrm{T}}=0 \cap X_{\mathrm{RT}} \cap X_{\mathrm{W}}=0 \cap X_{\mathrm{RW}}=0 \mid\right. \\
\left.T=t, W_{i}=w, W_{i j}=w_{i j} T_{j}=t_{j} C_{n}=c\right\}
\end{gathered}
$$

If $w \neq 0$, we have: 


$$
\begin{aligned}
& P\left\{X_{\mathrm{T}}=0 \cap X_{\mathrm{RT}}=0 \cap X_{\mathrm{W}}=0 \cap X_{\mathrm{RW}}=0\right. \\
& \left.\quad \mid T=t, W_{i}=w, W_{i j}=w_{i j} T_{j}=t_{j} C_{n}=c\right\}= \\
& \sum_{p 1=0}^{t_{j}}\left(1-S_{1}\right)^{p 1} S_{1}^{\left(t_{j}-p 1\right)} P\left\{n_{s} \in \bar{A}_{j i}(\alpha), s=1, . ., p 1\right\} \\
& \sum_{p 2=0}^{\left(t-1-t_{j}\right)}\left(1-S_{1}\right)^{p 2} S_{1}^{\left(t-1-t_{j}-p 2\right)} \\
& \sum_{p 3=0}^{w_{i j}}\left(1-S_{2}\right)^{p 3} S_{2}^{\left(w_{i j}-p 3\right)} P\left\{n_{s} \in \bar{A}_{j i}(\alpha), s=1, . ., p 3\right\} \\
& \sum_{p 4=0}^{\left(w-w_{i j}\right)}\left(1-S_{2}\right)^{p 4} S_{2}^{\left(w-w_{i j}-p 4\right)} \\
& P\left\{X_{\mathrm{R}}=0 \mid(p 1+p 2+p 3+p 4)\right\}
\end{aligned}
$$

where $S_{1}$ is the probability that an active node that is not hidden from $i$ and had the same performance as $i$ during the contention phase is eliminated:

$$
\begin{aligned}
S_{1} & =\sum_{k=0}^{w}\left(\begin{array}{l}
w \\
k
\end{array}\right) p_{h}^{k}\left(1-p_{h}\right)^{w-k} \\
& \sum_{z=1}^{(w-k+1)}\left(1-P\left\{T=z \mid C_{n}=(w-k+1)\right\}\right)
\end{aligned}
$$

and $S_{2}$ is the probability that an active node that is hidden from $i$ is eliminated:

$$
\begin{gathered}
S_{2}=\sum_{k 1=0}^{(w-1)} \sum_{k 2=0}^{t-1}\left(\begin{array}{c}
w-1 \\
k 1
\end{array}\right)\left(\begin{array}{c}
t-1 \\
k 2
\end{array}\right) \\
p_{h}^{(k 1+k 2)}\left(1-p_{h}\right)^{(w-1-k 1+t-1-k 2)} \\
\sum_{z=1}^{(w-k 1+t-1-k 2)}(1-P\{T=z \mid \\
\left.\left.C_{n}=(w-k 1+t-1-k 2)\right\}\right)
\end{gathered}
$$

Finally we can approximate $P\left\{X_{\mathrm{R}}=0 \mid p\right\}$ as:

$$
\begin{aligned}
& P\left\{X_{\mathrm{R}}=0 \mid p\right\}= \\
& \quad 1-\sum_{k=1}^{\min \{p,(T(n)-2-p)\}}\left(\begin{array}{c}
(T(n)-2-p) \\
k
\end{array}\right) \frac{p !}{(p-k) !} \\
& \quad\left(S_{3}\right)^{k}\left(1-S_{3}\right)^{(T(n)-2-p-k)} \\
& P\left\{n_{s} \in A_{i j}(\alpha), s=1, . ., k\right\}
\end{aligned}
$$

where $S_{3}$ is the probability that a specific active node that has determined channel access will transmit successfully to a specific node in $i^{\prime} s$ capture area for $j$ and that only this transmission will be detected by the receiver node. For nodes equally transmitting packets to all other nodes it is:

$$
S_{3}=p_{h}^{(k-1)}\left(1-p_{h}\right) \frac{1}{\sum_{k=0}^{m_{\mathrm{CP}}-1} T(n)-1}
$$

If $(w=0)$, we have the following special cases:

- If $(t=1)$ then:

$$
\begin{aligned}
& P\left\{X_{\mathrm{T}}=0 \cap X_{\mathrm{RT}}=0 \cap X_{\mathrm{W}}=0 \cap X_{\mathrm{RW}}=0\right. \\
& \left.\mid T=t, W_{i}=w, W_{i j}=w_{i j} T_{j}=t_{j} C_{n}=c\right\}=1
\end{aligned}
$$

- If $\left((t>1) \cap w_{j}=0\right)$ then:

$$
\begin{aligned}
& P\left\{X_{\mathrm{T}}=0 \cap X_{\mathrm{RT}}=0 \cap X_{\mathrm{W}}=0 \cap X_{\mathrm{RW}}=0\right. \\
& \left.\mid T=t, W_{i}=w, W_{i j}=w_{i j} T_{j}=t_{j} C_{n}=c\right\}= \\
& P\left\{X_{\mathrm{R}}=0 \mid t\right\}
\end{aligned}
$$

- If $\left((t>1) \cap w_{j} \neq 0\right)$, then:

$$
\begin{aligned}
& P\left\{X_{\mathrm{T}}=0 \cap X_{\mathrm{RT}}=0 \cap X_{\mathrm{W}}=0 \cap X_{\mathrm{RW}}=0\right. \\
& \left.\mid T=t, W_{i}=w, W_{i j}=w_{i j} T_{j}=t_{j} C_{n}=c\right\}= \\
& P\left\{n_{s} \in \bar{A}_{j i}(\alpha), s=1, . ., t_{j}\right\} \\
& P\left\{X_{\mathrm{RT}}=0 \mid t\right\}
\end{aligned}
$$

The overall expected renewal interval is:

$$
\begin{aligned}
& E\left(T_{\mathrm{S}}\right)= \\
& \sum_{n=0}^{m_{\mathrm{CP}}-1} P\left\{H_{n}=0\right\} \frac{\left(1-e^{-\left(G(n) E\left(T_{\mathrm{S}}\right)\right)}\right)}{\left(1-e^{-\left(G E\left(T_{\mathrm{S}}\right)\right)}\right.} E\left(T_{\mathrm{S}}(n)\right)
\end{aligned}
$$

Where $E\left(T_{\mathrm{S}}(n)\right)$ is the expected renewal interval after a packet of priority $n$ was transmitted. Given an initial value ( between $E\left(T_{\mathrm{S}}(0)\right)$ and $\left.E\left(T_{\mathrm{S}}\left(m_{p}-1\right)\right)\right)$ to the above equation, it will quickly converge([5]).

$E\left(T_{\mathrm{S}}(n)\right)$ is equal to: 


$$
\begin{aligned}
& E\left(T_{\mathrm{S}}(n)\right)=n P T+T_{\mathrm{TRANS}}+T_{\mathrm{ACK}} \\
& +E T \sum_{r=0}^{\left(m_{\mathrm{ES}}-1\right)}(r+1) \\
& \sum_{c=1}^{T(n)} P\left\{C_{n}=c\right\}^{*} P\left\{\max \left(X_{\mathrm{E}}\right)=r \mid C_{n}=c\right\} \\
& +Y T \sum_{s=0}^{\left(m_{\mathrm{YS}}-1\right)} s \sum_{c=1}^{T(n)} P\left\{C_{n}=c\right\}^{*} \\
& \sum_{j=1}^{c} P\left\{E=j \mid C_{n}=c\right\} P\left\{\min \left(X_{\mathrm{Y}}\right)=s \mid E=j\right\}
\end{aligned}
$$

and if $n$ is the lowest priority competing:

$$
\begin{aligned}
& E\left(T_{\mathrm{S}}(n)\right)=n P T+T_{\mathrm{TRANS}}+T_{\mathrm{ACK}} \\
& +E T\left(\sum_{r=0}^{\left(m_{\mathrm{ES}}-1\right)}(r+1)\right. \\
& \sum_{c=1}^{T(n)} P\left\{C_{n}=c\right\}^{*} P\left\{\max \left(X_{\mathrm{E}}\right)=r \mid C_{n}=c\right\} \\
& \left.+\left(m_{\mathrm{ES}}\right) P\left\{C_{n}=0\right\}^{*}\right) \\
& +Y T\left(\sum_{s=0}^{\left(m_{\mathrm{YS}}-1\right)} s \sum_{c=1}^{T(n)} P\left\{C_{n}=c\right\}^{*}\right. \\
& \sum_{j=1}^{c} P\left\{E=j \mid C_{n}=c\right\} P\left\{\min \left(X_{\mathrm{Y}}\right)=s \mid E=j\right\} \\
& \left.+\left(m_{\mathrm{YS}-1}\right) P\left\{C_{n}=0\right\}^{*}\right)
\end{aligned}
$$

where $P\left\{C_{n}=c\right\}^{*}$ is the probability of any $c$ nodes being active (no specific node $i$ is required).

where PT, ET and YT are the time intervals of a priority, elimination and yielding slots, and EP and $\mathrm{YP}$ are the probabilities of bursting during an elimination slot and yielding during a yielding slot, $T_{\text {TRANS }}$ is the time to transmit a packet and $T_{\mathrm{ACK}}$ is the time to transmit an acknowledgment, and

for $c>0$ :

$$
\begin{gathered}
P\left\{\max \left(X_{\mathrm{E}}\right)=r \mid C_{n}=c\right\}= \\
\sum_{m=1}^{c} C_{m}^{c} P\left\{X_{\mathrm{E}}=r\right\}^{m} P\left\{X_{\mathrm{E}}<r\right\}^{(c-m)} \\
P\left\{\min \left(X_{\mathrm{Y}}\right)=s \mid C_{n}=c\right\}= \\
\sum_{m=1}^{c} C_{m}^{c} P\left\{X_{\mathrm{Y}}=s\right\}^{m} P\left\{X_{\mathrm{Y}}>s\right\}^{(c-m)}
\end{gathered}
$$

$$
\begin{aligned}
& \text { for } c=0 \text { and } r=m_{\mathrm{ES}}-1 \text { : } \\
& \qquad \begin{aligned}
& P\left\{\max \left(X_{\mathrm{E}}\right)=r \mid C_{n}=c\right\}=1 \\
& \text { and for } c= 0 \text { and } r=m_{\mathrm{YS}}-1: \\
& P\left\{\min \left(X_{\mathrm{Y}}\right)=s \mid C_{n}=c\right\}=1
\end{aligned}
\end{aligned}
$$

\section{Numerical Results}

The numerical results are now presented. We are interested in the total throughput $S$ of the system versus the total load $G$ for various values of the probability $p_{\mathrm{h}}$ that two nodes are hidden from each other and various values of the capture parameter $a$. This will determine the system's performance. In order to gather the necessary data, two software packages were developed, a simulation program and a program for calculating the theoretical data.

The protocol parameters used are: priority slot duration $i_{\mathrm{PS}}=256$ bits, $p_{\mathrm{E}}=0.5$, elimination slot duration $i_{\mathrm{ES}}=256$ bits, $p_{\mathrm{Y}}=0.9$, yield slot duration $i_{\mathrm{YS}}=64$ bits, constant packet size 14880 bits and acknowledgment packet size 512 bits. The channel bandwidth is $23529 \mathrm{bits} / \mathrm{sec}$. Our WLAN consists of 25 nodes that were placed randomly in space.

The coordinates of the nodes were chosen randomly and are normalized to belonging inside a circle of radius $1:(0.28,0.70),(0.38,-0.21),(0.89,-0.21),(0.36,-$ $0.65),(-0.09,0.08),(-0.74,0.40),(-0.26,0.03),(0.56,0.00)$, $(0.62,-0.94),(0.79,0.78),(-0.08,0.34),(0.59,0.99),(0.94,-$ $0.62), \quad(0.73,0.41), \quad(0.76,-0.11), \quad(-0.37,0.55), \quad(0.17,-$ $0.16),(0.67,0.71),(0.57,-0.20),(0.68,-0.16),(-0.47,0.10)$, $(0.42,0.63),(0.43,0.08),(-0.28,-0.21),(0.52,-0.92)$.

For simplicity but with no loss in generality we assumed all nodes transmitting the same amount of load and equally to all other nodes. We are also assuming all nodes to be of the same priority level, thus no higher priority nodes exist. After studying the performance of this highest priority level we can also draw from this study conclusions about the performance of lower priority levels, since their performance will be decreased by a factor of $P\left\{H_{n}=0\right\}$, that only depends on the total load coming from nodes of higher priority.

The simulation data and the analytical data are practically identical, thus our approximation (as expected) was a very good one. For this reason, both data are represented by a single curve for each pair of parameters $p_{\mathrm{h}}$ and $\alpha$.

In Figures 4 and 5 we see the effect on performance of the variation of the capture parameter $\alpha$ for a specific probability $p_{\mathrm{h}}$. In Figure 4 we have chosen $p_{\mathrm{h}}$ equal to 0.01 and in Figure 5 we have chosen $p_{\mathrm{h}}$ equal to 0.05 . As we can 
see performance decreases as the possibility of capture becomes less likely ( $\alpha$ increases). This decrease, as expected, is bigger for the higher $p_{\mathrm{h}}$, since in this case the effect of hidden nodes on performance is greater.

In Figures 6 and 7 we see the effect on performance of the variation of the probability $p_{\mathrm{h}}$ for specific values of $\alpha$. In Figure 6 we have chosen $\alpha=1.2$. This is a relatively small value for $\alpha$ and indicates a high presence of the phenomenon of capture. In Figure 7 we have chosen $\alpha=10.0$. This is a relatively high value for $\alpha$ and is actually the case of no presence of the phenomenon of capture. As we can see the performance decreases as the probability $p_{\mathrm{h}}$ increases. The decrease is bigger for the higher $\alpha$, since there is no benefit to the performance from the capture effect.

These results show clearly the importance of hidden nodes and capture on the performance of the HIPERLAN CAC Layer protocol.

\section{Conclusion}

We have presented a brief outline of the HIPERLAN CAC (MAC) layer protocol and discussed in detail the analysis of the protocol performance under asynchronous traffic. We argued and presented the efficiency of an approximation in calculating the probability of a successful transmission between two nodes $i$ and $j, P_{i j}$. Finally we presented the numerical results of both simulation and analysis that where practically identical. Our future work involves studying the HIPERLAN CAC Layer performance under time-bounded traffic.

\section{References}

[1] G. Anastasi, L. Lenzini, and E. Mingozzi. Stability and performance analysis of hiperlan. Proceedings of the IEEE Infocom, 1998.

[2] S. Chevrel, A.H. Aghvami, H.Y. Lach, and L. Taylor. Analysis and optimasation of the hiperlan channel access contention scheme. Wireless Personal Communications, 4(May):27-39, 1996.

[3] H. Chhaya and S. Gupta. Performance evaluation of asynchronous data transfer methods in 802.11 MAC protocol. Wireless Networks, 3, 1997.

[4] H. S. Chhaya and S. Gupta. Performance of asynchronous data transfer medhods in ieee 802.11 MAC protocol. IEEE Personal Communications Magazine, 3(5):8-15, 1996.

[5] Edwin K. P. Chong and Stanislav H. Zak. An Introduction To Optimization. John Wiley and Sons, Inc, 1996.
[6] C. Coutras, S. Gupta, and N. Shroff. Scheduling of real time traffic over IEEE 802.11 WLANs. Wireless Networks, To appear, 1999.

[7] European Telecommunication Standards Institute, 650 Route des Lucioles, Sophia Antipolis, Valbonne, France. HIgh PErformance Radio Local Area Network Type 1: functional specification, pr ets 300652 edition, May 1996.

[8] K. Fu, Y.J. Guo, and S.K. Barton. Performance of the ey-npma protocol. Wireless Personal Communications, 4(May):41-50, 1996.

[9] Philippe Jacquet, Pascale Minet, Paul Muhlethaler, and Nicolas Rivierre. Data transfer for hiperlan. Wireless Personal Communications, 4(May):65-80, 1996.

[10] Philippe Jacquet, Pascale Minet, Paul Muhlethaler, and Nicolas Rivierre. Priority and collision detection with active signaling - the channel access mechanism of hiperlan. Wireless Personal Communications, 4(May):11-25, 1996.

[11] Richard O. LaMaire, Arvind Krishna, Pravin Bhagwat, and James Panian. Wireless lans and mobile networking: Standards and future directions. IEEE Communications Magazine, (August):86-94, 1996.

[12] W. Melody Moh, Dongming Yao, and K. Makki. Wireless lan: Study of hidden-terminal effect and multimedia support. Proceedings of the IEEE Infocom, pages 422-431, 1998. 


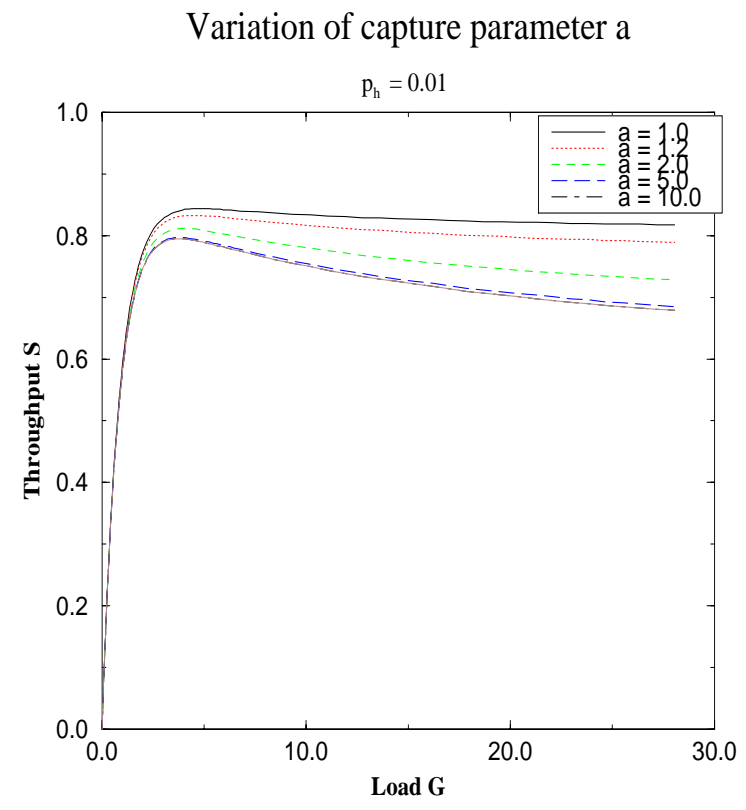

Figure 4. capture parameter variation

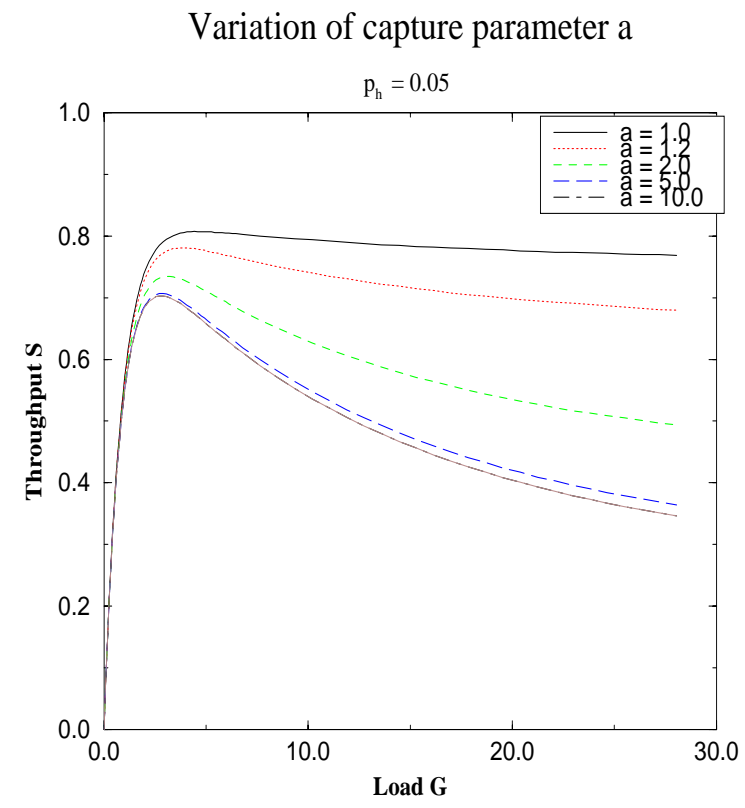

Figure 5. capture parameter variation

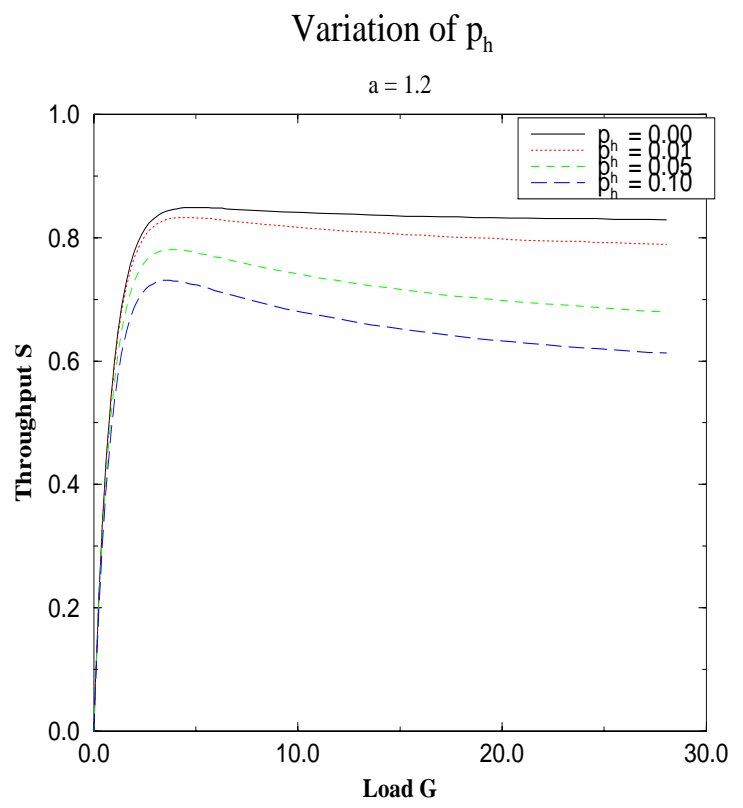

Figure 6. $p_{h}$ variation

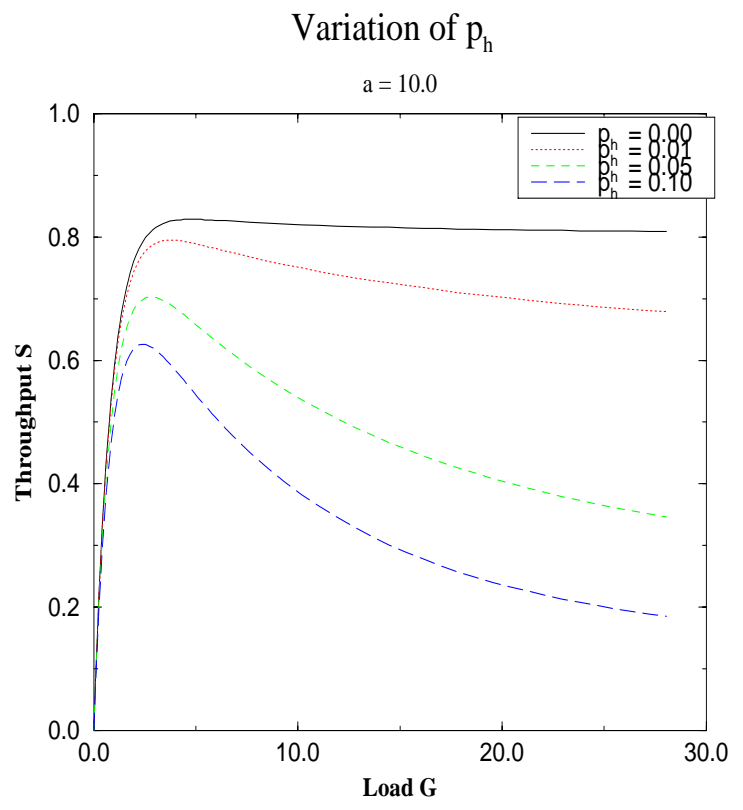

Figure 7. $p_{h}$ variation 